\title{
An Experimental Study of Retarder MRF Performance Testing Device
}

\author{
Qixiao Jie ${ }^{1, a}$, Anyong Dong ${ }^{2, b}$, Bao $\mathrm{Yu}^{3, \mathrm{c}}$, Lvde Gang $^{4, \mathrm{~d}}$ \\ ${ }^{1}$ College of Automobile and Traffic Engineering, Heilongjiang Institute Of Technology, \\ Harbin, 150050, China \\ ${ }^{2}$ College of Automobile and Traffic Engineering, Heilongjiang Institute Of Technology, \\ Harbin,150050, China \\ ${ }^{3}$ College of Automobile and Traffic Engineering, Heilongjiang Institute Of Technology, \\ Harbin,150050, China \\ ${ }^{4}$ College of Automobile and Traffic Engineering, Heilongjiang Institute Of Technology, \\ Harbin,150050, China \\ aemail: qxj725@sina.com, bemail:510197353@qq.com, 'email: 365270930@qq.com, demail: \\ 502727779@qq.com
}

Keywords: Magneto-Rheological Fluid; Retarder;Test Device; Experimental Study

\begin{abstract}
The paper designs a performance test device to detect the performance of the magnet-orheological retarder at different temperatures,different magnetic fields and different speeds, and completed the experimental study. The device structure has the characteristic of simple, reliable drive, convenient and high precision, and test the rheological properties of magneto-rheological fluid under different temperatures, different magnetic field strength.The test device has advantage of small residual magnetism, test data accurate,good repeatability of test data and low power consumption.
\end{abstract}

\section{Introduction}

Magnetorheological fluid (MRF) as a controllable fluid is a suspension from the mixture of high-permeability and low-hysteresis tiny soft magnetic particles and non-magnetic fluid. The suspension demonstrates low-viscosity Newtonian fluid characteristics under zero magnetic field conditions, while high-viscosity and low-flowability characteristics in strong magnetic fields. Due to the rapid magnetorheological response, easy control and other features of MRF, MRF devices have been gradually applied to vehicle vibration control, mechanical power transmission, precision machining of workpieces and other industrial fields. With the application of MRF devices, it is particularly important to test the rheological properties of MRF of different components under different conditions. Testing the rheological properties demonstrated by different magnetorheological fluids under different conditions can provide strong technical support for the research and development of new MRF devices [1] [2] [3].

\section{Structural Composition and Working Principle of Retarder MRF Performance Testing Device}

(1) Basic Composition of the Test Bench

Magnetorheological fluid test bench is a test system used to test magnetorheological fluids. In this system, different magnetic field effects are generated by the generator on the MRF in the working chamber, and the MRF demonstrates different magnetorheological properties and different damping torques under the effect of the magnetic fields. The driving force generated by the driving device will be reduced due to the magnetic resistance effect, and the torque generated by the MRF can be measured with the torque measuring device. The test bench is shown in Figure1.

The MRF test bench consists of a driving device, a detection device and a generator, wherein the driving device is composed of the drive shaft externally connected to a speed-controllable motor; 
the detection device consists of a sensor holder, sensor spacer sleeve, single thrust bearing and upper cover of the sensor; the generator is composed of lower cover of the test tank, spacer sleeve of the test tank, upper cover of the rotation disk test tank, circular magnet yoke of the nylon inner sleeve and winding core, as shown in Figure2.

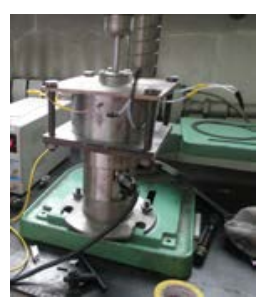

Figure 1 MRF test bench

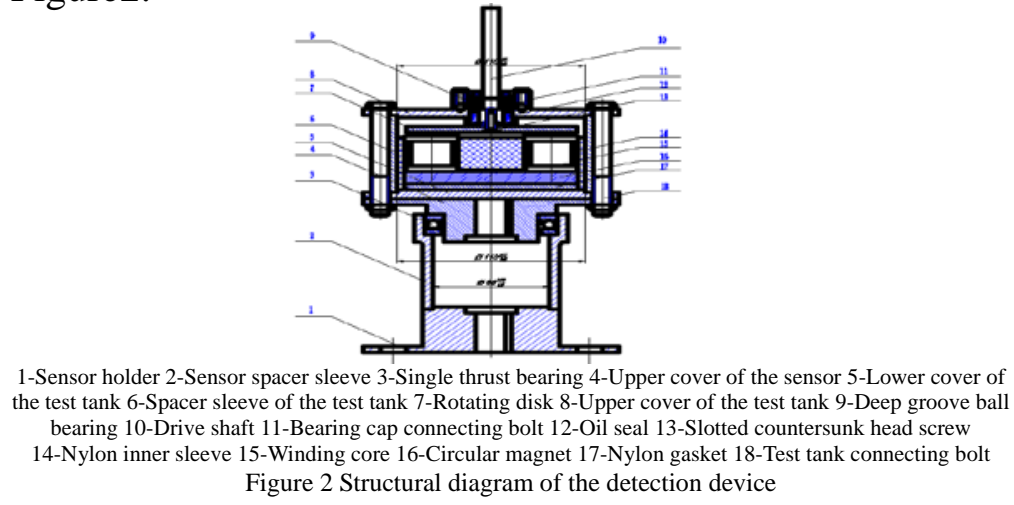

Figure 2 Structural diagram of the detection device

(2) Working Principle of the Detection Device

The gap between end faces of the rotating disk and the winding core of the detection device is the working chamber for testing rheological properties of the MRF. The size of the working chamber can be adjusted through the gasket between upper cover and spacer sleeve of the test tank so as to test rheological properties of the MRF in different gaps. The magnetic field is generated by six coils. The six coils are arranged circumferentially, adjacent coils are contrarily wound, and the magnetic field generated by any coil forms a loop through the two adjacent coils. As a result, the MRF is subject to a part of forward magnetic field and a part of reverse magnetic field. When the magnetic field disappears, under the effect of inertia of the rotating disk, the MRF under the simultaneous effects of the forward and reverse fields rapidly flows and fuses to neutralize the remaining magnet, which can help the MRF to quickly restore its original state, eliminate the effects of the coercive force on the results of repeated tests, and overcome the disadvantage of previous MRF testing instruments. In the case of previous MRF testing instruments, after the first test, transmission torque of the MRF under the influence of residual magnetization cannot be quickly reduced or change the direction of action of the magnetic field, and the presence of coercive force will affect the results of next test.

The excitation mode uses three forward excitation coils for simultaneous power supply[4] [5]. Reverse excitation coils can also be used for power supply. As the number of turns, diameter size and material of the coils are exactly the same, the magnetic flux density can be reduced by half. For either forward or reverse coil, the current intensity can be controlled by using a DC power supply instrument, and the operation is simple, quick and precise. The excitation coils are mounted inside the nylon inner sleeve and wound on the core, and a magnet yoke specifically for magnetic conduction is installed below. Both the core and the yoke are made of high-permeability magnet steel, and thus can effectively reduce iron loss. The lower part of the yoke is equipped with a nylon bush, which can prevent leakage of the magnetic field lines, and ensure action of exciting fields inside the MRF, so that the test results are more accurate and power consumption is lower.

Speed of the input shaft is adjustable, the drive shaft is hollow, and a thermocouple is stuck onto the rotating disk. The MRF temperature can be measured through slip rings, and thus MRF performance at different temperatures and different input shaft speeds under different magnetic field strengths can be further obtained[6] [7].

\section{Experimental Process and Data Analysis}

(1) Experimental Process

The drive shaft is driven by the motor to operate and send the connected rotating disk rotating. The rotating disk is put into the MRF, and together with the MRF between the end faces of the winding core, produces a reluctance torque under the effect of the magnetic field. The reluctance 
torque is transmitted to the spacer sleeve of the test tank through the winding core, circular magnet yoke and nylon inner sleeve, and then the spacer sleeve of the test tank transmits the reluctance torque to the upper cover of the sensor which is connected to the sensor through a flat key. Then the sensor can measure the reluctance torque. The experimental process is shown in Figure3.In this device, the gap between end faces of the rotating disk and the winding core is the working chamber for testing rheological properties of the MRF. The size of the working chamber can be adjusted through the gasket between cover and spacer sleeve of the test tank so as to test rheological properties of the MRF in different gaps.

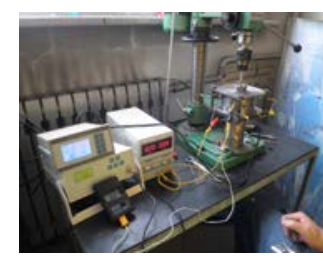

Fig.3 Experimental process

(2) Experimental Data Analysis

In the experiment, the effects of different input currents, different working temperatures and different working speeds on properties of the MRF were studied and analyzed. During the experimental process, main experimental instruments used included MRF detection device, DC regulated power supply, Oe magnetic field meter and speed \& torque measuring instrument.

1)Effect of currents

The detection device was debugged, including analysis and determination of the testing chamber gap, amount of MRF added, magnetic coil current direction and magnetic field direction. The form of alternate setting of $\mathrm{N}$ and $\mathrm{S}$ poles was adopted for the internal magnetic field direction. The magnetic field intensity generated around the MRF was the maximum, so the test data were all obtained from tests by using this connection. DC regulated power supply was used to control the input current which increased from small to large. Data were read on the magnetic field meter and torque measuring instrument and recorded. Relationship between currents and magnetic field changes is shown in Figure4, and the effect of current changes on reluctance torque is shown in Figure5. It can be seen from the change curves that as the current increases, the magnetic field intensity increases, basically showing a linear change relationship; the effect of current on reluctance torque shows a trend of slowly increase first, then rapid increases, and eventually extremely rapid increase.

2)Effect of temperature

The effect of MRF temperature on the output torque of MRF was studied through an experiment. In this test, the instruments used included MRF detection device, DC regulated power supply, temperature gauge and speed \& torque measuring instrument. The test was conducted at room temperature first, and then the input current was changed to measure and record the output torque. After operation reached the testing temperature, the current was changed again to measure and record the output torque. By using the same method, the temperature range was repeatedly changed to measure and compare the output torque, and the change relationships shown in Figure6, Figure7 and Figure8 were obtained. In this test, three groups of different torque changes of the MRF at three (low, medium and high) different temperatures were tested. It could be seen from the curve change trends that within different temperature ranges, changes in the reluctance torque of the MRF were consistent with the change trend of the current, increasing as the current increased. However, the torque showed a slightly declining trend as temperature increased, but the trend was not obvious.

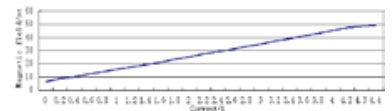

Fig.4 Relationship between current and magnetic field

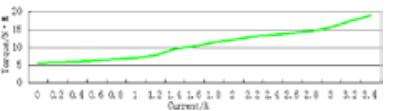

Fig.5 Effect of current on reluctance torque

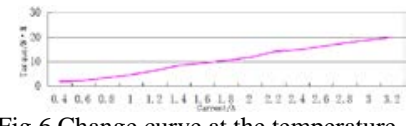

Fig.6 Change curve at the temperature of $22-26^{\circ} \mathrm{C}$

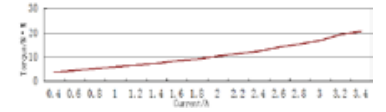

Fig.7 Change curve at the temperature of $47-50^{\circ} \mathrm{C}$

3) Effect of speed 
In this test, torque changes of the MRF at different speeds were detected by changing the speeds of the driving device, and changes in output torque at different speeds were studied. Instruments used included MRF detection device, DC regulated power supply, torque measuring instrument and speed \& torque measuring instrument. Speed of the motor was controlled at $500 \mathrm{r} / \mathrm{min}$ or $1500 \mathrm{r} / \mathrm{min}$ for the test. DC regulated power supply was used to successively increase the current. Output data were respectively read on the torque measuring instrument and recorded, and relationship curves as shown in Figure9 and Figure 10 were obtained. It could be seen from the curve changes that when the MRF rotated at a high speed, in the early stage of current increase, the increase in reluctance torque was very slow, but with further increase in the current, reluctance torque increased rapidly. In case of high-speed operation, to achieve a safe slowing effect, current input must be increased in the early stage of braking so as to obtain reliable braking effect.

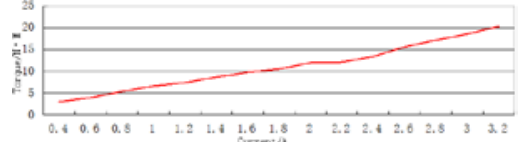

Fig.8 Change curve at the temperature of $64-67^{\circ} \mathrm{C}$

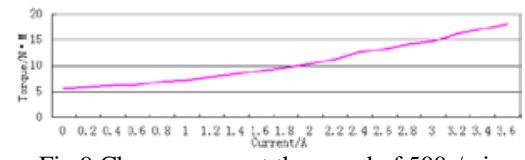

Fig.9 Change curve at the speed of $500 \mathrm{r} / \mathrm{min}$

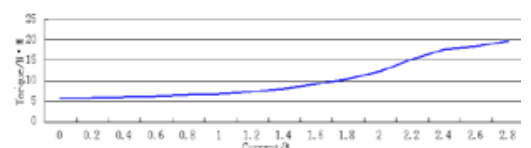

Fig.10 Change curve at the speed of $1500 \mathrm{r} / \mathrm{min}$

\section{Conclusion}

This paper, by experimentally studying the MRF detection device developed, has obtained the change relationships of MRF reluctance torque under different currents, different magnetic field intensities, different working temperatures and different operating speeds, and the following conclusions can be drawn from the change relationships:

(1) MRF increases with increasing current and increasing magnetic field. The stronger the magnetic field, the greater the reluctance torque generated, and the greater the braking torque of the MRF retarder.

(2) When the input current remains unchanged, the reluctance torque demonstrated by the MRF shows a declining trend as the temperature rises, and the declining trend is not obvious when the input current is small, but as the input current increases, the declining trends gets more obvious.

(3) At the same input current, the MRF reluctance torque increases with increasing speed, but at high speed and small input current, the increasing trend of the reluctance torque is slower than at low speeds; after the input current reaches a certain value, the increasing trend of the reluctance torque gets faster. To ensure reliable braking effect of the MRF retarder at high speeds, large current must be input in the early stage of braking, which has also provided a theoretical basis for further research and development of MRF retarders.

\section{Acknowledgement}

In this paper, the research was sponsored by the Dr Fund Project of Heilongjiang Institute Of Technology (Project No.2011BJ04).

\section{References}

[1] Tianzu Zhi, Houyou Fu. Calculation model research of MRF yield stress [J]. Journal of China Mining University. 2012 (02):25-29.

[2] Dongzi Xin, Lifei Hu. Test research review of MRF yield stress [J]. Journal of Information Science \& Technology. 2011 (22):145-148.

[3] Tang Long, Yue En. Temperature characteristic study of MRF [J]. Journal of Functional Materials. 2011 (6):67-72.

[4] Tianzu Zhi. Research of MRF and Transmission Technology [D]. China Mining University, 2012.

[5] Yicheng Jian, Pengxiang He, Sun Hu. Analysis of MRF Microstructure magnetization and mechanical characteristics based on the FEM [J]. Journal of Functional Materials. 2011 (8):32-37. 
[6] Zekeriya Parlak,Tahsin Engin.Time-dependent CFD and quasi-static analysis of magnetorheological fluid dampers with experimental validation[J]. International Journal of Mechanical Sciences.2012.

[7] Lifei Hu,Guochu Wen,Dongzi Xin. Influence factors research of MRF stability[J].Journal of Information Science \& Technology. 2012 (4):111-116. 